\title{
Research Article \\ Lebesgue Measurability of Separately Continuous Functions and Separability
}

\author{
V. V. Mykhaylyuk \\ Received 4 September 2006; Accepted 22 April 2007 \\ Recommended by Peter Johnson
}

A connection between the separability and the countable chain condition of spaces with $L$-property (a topological space $X$ has $L$-property if for every topological space $Y$, separately continuous function $f: X \times Y \rightarrow \mathbb{R}$ and open set $I \subseteq \mathbb{R}$, the set $f^{-1}(I)$ is an $F_{\sigma}$-set) is studied. We show that every completely regular Baire space with the $L$-property and the countable chain condition is separable and constructs a nonseparable completely regular space with the $L$-property and the countable chain condition. This gives a negative answer to a question of M. Burke.

Copyright (c) 2007 V. V. Mykhaylyuk. This is an open access article distributed under the Creative Commons Attribution License, which permits unrestricted use, distribution, and reproduction in any medium, provided the original work is properly cited.

\section{Introduction}

A function $f: X \rightarrow \mathbb{R}$ defined on a topological space $X$ is called a first Baire class function if there exists a sequence $\left(f_{n}\right)_{n=1}^{\infty}$ of continuous functions $f_{n}: X \rightarrow \mathbb{R}$ which converges pointwise to $f$ on $X$; and a first Lebesgue class function if $f^{-1}(G)$ is an $F_{\sigma}$-set for every open set $G \subseteq \mathbb{R}$. Standard reasons (see [1, page 394]) show that every first Baire class function is a first Lebesgue class function.

Investigations of Baire and Lebesgue classifications of separately continuous functions were started by Lebesgue in [2] and were continued in papers of many mathematicians (see [3]).

We say that a topological space $X$ has the B-property (the L-property) if for every topological space $Y$ each separately continuous function $f: X \times Y \rightarrow \mathbb{R}$ is a first Baire class function (a first Lebesgue class function).

It is known $[4,5]$ that any topological space $X$ has the $B$-property (the $L$-property) if and only if the evaluation function $c_{X}: X \times C_{p}(X) \rightarrow \mathbb{R}, c_{X}(x, y)=y(x)$ is a first Baire 
class function (a first Lebesgue class function), where $C_{p}(X)$ means the space of continuous on $X$ functions with the pointwise convergence topology.

Baire and Lebesgue classifications of separately continuous function were investigated in [6]. In particular, it was shown in [6] that any completely regular space $X$ with the $B$-property and the countable chain condition is separable (topological space $X$ has a countable chain condition (CCC) if every system of disjoint open-in- $X$ sets is at most countable). In this connection the following question arose in [6, Problem 4.6].

Question 1. Is every completely regular space $X$ with the $L$-property and the countable chain condition a separable space?

In this paper, we show that if a space $X$ is a Baire space, then Question 1 has a positive answer and construct an example which gives a negative answer to the question in general case.

\section{Density of Baire spaces with the $L$-property}

The minimal cardinal $\mathrm{N} \geq \mathrm{N}_{0}$ for which any system of disjoint open in a topological space $X$ sets has the cardinality at most $\kappa$ is called a Souslin number of $X$ and is denoted by $c(X)$. Note that the countable chain condition of $X$ means that $c(X)=\aleph_{0}$. It is easy to see that $c(X) \leq d(X)$, where $d(X)$ is the density of $X$.

The following result implies that for a Baire space $X$ Question 1 has a positive answer.

Theorem 2.1. Let $X$ be a completely regular Baire space with the L-property. Then $c(X)=$ $d(X)$.

Proof. Since the evaluation function $c_{X}$ is a first Lebesgue class function, the set $E=$ $\{(x, y): y(x)=0\}$ is a $G_{\delta}$-set in $X \times Y$, where $Y=C_{p}(X)$. Choose a sequence $\left(W_{n}\right)_{n=1}^{\infty}$ of open-in- $X \times Y$ sets $W_{n}$ such that $E=\bigcap_{n=1}^{\infty} W_{n}$. Denote by $y_{0}$ the null-function on $Y$. For every $n \in \mathbb{N}$ and an $x \in X$ find open neighborhoods $U(x, n)$ and $V(x, n)$ of $x$ and $y_{0}$ in $X$ and $Y$, respectively, such that $U(x, n) \times V(x, n) \subseteq W_{n}$.

Fix an $n \in \mathbb{N}$ and show that there exists a set $A_{n} \subseteq X$ with $\left|A_{n}\right| \leq c(X)=\mathcal{N}$ such that the open set $G_{n}=\bigcup_{x \in A_{n}} U(x, n)$ is dense in $X$. Consider a system $\mathcal{U}$ of all open-in- $X$ nonempty sets $U$ such that $U \subseteq U(x, n)$ for some $x \in X$ and choose a maximal system $\mathcal{U}^{\prime} \subseteq \mathcal{U}$ which consists of disjoint sets. It is clear that $\left|\mathcal{U}^{\prime}\right| \leq \kappa$. For every $U \in \mathcal{U}^{\prime}$ find an $x=x(U) \in X$ such that $U \subseteq U(x, n)$ and put $A_{n}=\left\{x(U): U \in U^{\prime}\right\}$. Then $\left|A_{n}\right| \leq\left|u^{\prime}\right| \leq$ $\kappa$. Besides, it follows from the maximality of $U^{\prime}$ that $G_{n}$ is dense in $X$.

Since $X$ is a Baire space, the set $X_{0}=\bigcap_{n=1}^{\infty} G_{n}$ is dense in $X$. For every $n \in \mathbb{N}$ and $x \in X$ choose a finite set $B(x, n) \subseteq X$ such that $y \in V(x, n)$ for each $y \in Y$ with $\left.y\right|_{B(x, n)}=$ $\left.y_{0}\right|_{B(x, n)}$. Put $B=\bigcup_{n \in \mathbb{N}} \bigcup_{x \in A_{n}} B(x, n)$. Note that $|B| \leq \aleph_{0} \cdot \kappa=\kappa$.

Show that $B$ is dense in $X$. Since $X$ is a completely regular space, it is enough to prove that $y_{0}$ is a unique continuous on $X$ function which equals to 0 at every point from $B$. Let $y \in Y$ be a function such that $y(b)=0$ for every $b \in B$. Fix a point $x \in X_{0}$ and an integer $n \in \mathbb{N}$. Find $a \in A_{n}$ such that $x \in U(a, n)$. Then $B(a, n) \subseteq B$ implies $y \in V(a, n)$. Therefore, $(x, y) \in W_{n}$. Thus $X_{0} \times\{y\} \subseteq \bigcap_{n=1}^{\infty} W_{n}=E$, that is, $y(x)=0$ for every $x \in X_{0}$. Hence $y=y_{0}$ because $X_{0}$ is dense in $X$.

Thus $d(X) \leq|B| \leq c(X)$. Therefore, $c(X)=d(X)$. 
COROLlARY 2.2. Every completely regular Baire space with the L-property and the countable chain condition is a separable space.

\section{Nonseparable spaces with the $L$-property and CCC}

The following notion was introduced in [4], where some properties of spaces with the $B$-property were studied.

A topological space $X$ with a topology $\tau$ is called quarter-stratifiable if there exists a function $g: \mathbb{N} \times X \rightarrow \tau$ such that

(i) $X=\bigcup_{x \in X} g(n, x)$ for every $n \in \mathbb{N}$;

(ii) if $x \in g\left(n, x_{n}\right)$ for each $n \in \mathbb{N}$, then $x_{n} \rightarrow x$.

The following result follows from [7, Proposition 2.1].

Proposition 3.1. Every quarter-stratifiable space X has the L-property.

A topological space $X$ is called $\sigma$-discrete if there exists an increasing sequence $\left(X_{n}\right)_{n=1}^{\infty}$ of closed discrete subspaces $X_{n}$ of $X$ such that $X=\bigcup_{n=1}^{\infty} X_{n}$.

Proposition 3.2. Every $\sigma$-discrete space is a quarter-stratifiable space.

Proof. Let $\left(X_{n}\right)_{n=1}^{\infty}$ be an increasing sequence of closed discrete subspaces $X_{n}$ of $X$ such that $X=\bigcup_{n=1}^{\infty} X_{n}$. For every $n \in \mathbb{N}$ and $x \in X_{n}$ denote by $U(x, n)$ an open-in- $X$ neighborhood of $x$ such that $U(x, n) \cap X_{n}=\{x\}$. We define a function $g: \mathbb{N} \times X \rightarrow \tau$, where $\tau$ is the topology of $X$, by $g(x, n)=U(x, n)$ if $x \in X_{n}$ and $g(x, n)=X \backslash X_{n}$ if $x \notin X_{n}$. It is easy to see that $g$ satisfies (i) and (ii).

Show now that Question 1 has a negative answer.

THEOREM 3.3. There exists a completely regular nonseparable space with the L-property and with the countable chain condition.

Proof. Let $\Gamma_{0}$ be a set with $\left|\Gamma_{0}\right| \geq \kappa_{1}$, let $\left(a_{n}\right)_{n=1}^{\infty}$ be a sequence of distinct points $a_{n} \notin \Gamma_{0}$, $\Gamma_{n}=\Gamma_{0} \cup\left\{a_{k}: 1 \leq k \leq n\right\}$, and let $\mathscr{A}_{n}$ be a system of all subsets $A \subseteq \Gamma_{n-1}$ such that $|A|=$ $n$. Denote by $X_{n}$ a set of all function $x \in\{0,1\}^{\Gamma}$ such that $x=\chi_{A \cup\left\{a_{n}\right\}}$ for some $A \in \mathscr{A}_{n}$, where $\chi_{B}$ means the characteristic function of $B$, and put $X=\bigcup_{n=1}^{\infty} X_{n}$.

Show that $X$ is a $\sigma$-discrete space. For every $n \in \mathbb{N}$ put $Y_{n}=\bigcup_{k=1}^{n} X_{k}$. Fix an integer $n \in \mathbb{N}$ and for each $1 \leq k \leq n$ put $G_{k}=\left\{x \in X: x\left(a_{k}\right)=1, x\left(a_{i}\right)=0, k<i \leq n\right\}$. It is easy to see that $G_{k} \cap Y_{n}=X_{k}$. Since all spaces $X_{k}$ are discrete, $Y_{n}$ is discrete in $X$ too. Besides, $Y_{n}$ is closed in $X$. Thus, $X$ has the $L$-property by Propositions 3.1 and 3.2.

Note that $X$ is dense in $Y=\{0,1\}^{\Gamma}$. Indeed, let $A \subseteq \Gamma$ be a finite set and $y: A \rightarrow\{0,1\}$. Choosing $n \geq|A|$ with $A \subseteq \Gamma_{n}$ find $x \in X_{n+1}$ such that $\left.x\right|_{A}=y$. Then $c(X)=\aleph_{0}$ since $c(Y)=\kappa_{0}$ and $X$ is dense in $Y$.

It remains to note that $X$ is nonseparable because for every separable subspace $Z$ of $X$ there exists a countable set $B \subseteq \Gamma$ such that $z(\gamma)=0$ for every $\gamma \in \Gamma \backslash B$.

This example shows that there exists a quarter-stratifiable space which has not the $B$ property. Thus, Proposition 3.1 cannot be generalized for spaces with the $B$-property. 


\section{International Journal of Mathematics and Mathematical Sciences}

A family $\left(A_{i}: i \in I\right)$ of sets $A_{i}$ is called pointwise finite if $\bigcap_{i \in J} A_{i}=\varnothing$ for each infinite set $J \subseteq I$. A cardinal

$$
p(X)=\sup \{|\mathscr{A}|: \mathscr{A} \text { is a pointwise finite family of nonempty open-in- } X \text { sets }\}
$$

is called a point-finite cellularity of a topological space $X$. Clearly $c(X) \leq p(X)$. Besides, it is known that $p(X)=c(X)$ for each Baire space $X$. Therefore, the following question arises naturally from Theorem 2.1 and the fact that $p(X)=|\Gamma|>\aleph_{0}$ for the space $X$ from Theorem 3.3.

Question 2. Is every completely regular space $X$ with the $L$-property and $p(X)=\mathrm{\aleph}_{0}$ a separable space?

\section{References}

[1] K. Kuratowski, Topology. Vol. I, Mir, Moscow, Russia, 1966.

[2] H. Lebesgue, "Sur l'approximation des fonctions," Bulletin des Sciences Mathématiques, vol. 22, pp. 278-287, 1898.

[3] V. K. Maslyuchenko, O. V. Maslyuchenko, V. V. Mykhaylyuk, and O. V. Sobchuk, "Paracompactness and separately continuous mappings," in General Topology in Banach Spaces, pp. 147-169, Nova Science Publishers, Huntington, NY, USA, 2001.

[4] T. O. Banakh, "(Metrically) quarter-stratifiable spaces and their applications in the theory of separately continuous functions," Matematichnī Studīi, vol. 18, no. 1, pp. 10-28, 2002.

[5] M. R. Burke, "Borel measurability of separately continuous functions," Topology and Its Applications, vol. 129, no. 1, pp. 29-65, 2003.

[6] M. R. Burke, "Borel measurability of separately continuous functions. II," Topology and Its Applications, vol. 134, no. 3, pp. 159-188, 2003.

[7] O. O. Karlova, "The first functional Lebesgue class and Baire classification of separately continuous mappings," Naukovyj Visnyk Chernivets'kogo Universytetu. Matematyka, vol. 191-192, pp. 52-60, 2004 (Ukrainian).

V. V. Mykhaylyuk: Department of Mathematical Analysis, Chernivtsi National University, Kotsjubyns'koho 2, 58012 Chernivtsi, Ukraine

Email address: mathan@chnu.cv.ua 


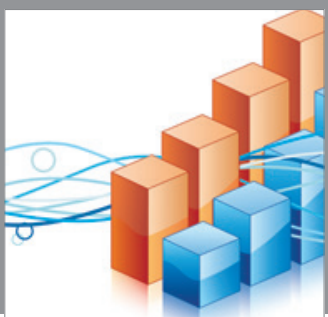

Advances in

Operations Research

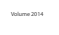

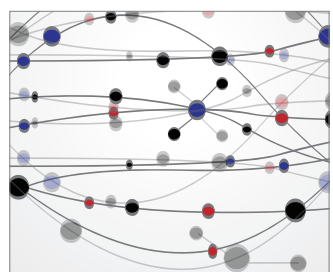

\section{The Scientific} World Journal
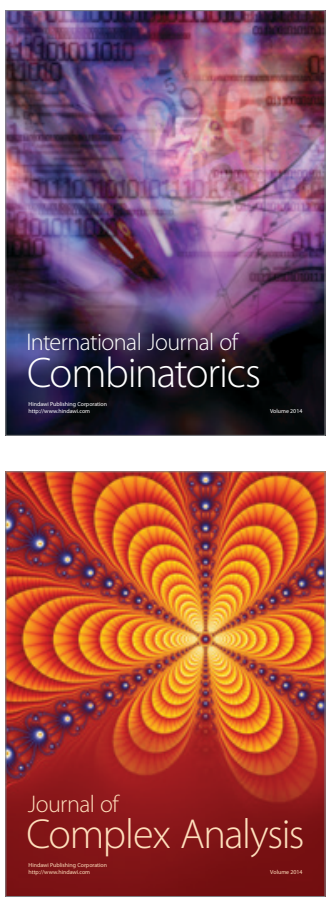

International Journal of

Mathematics and

Mathematical

Sciences
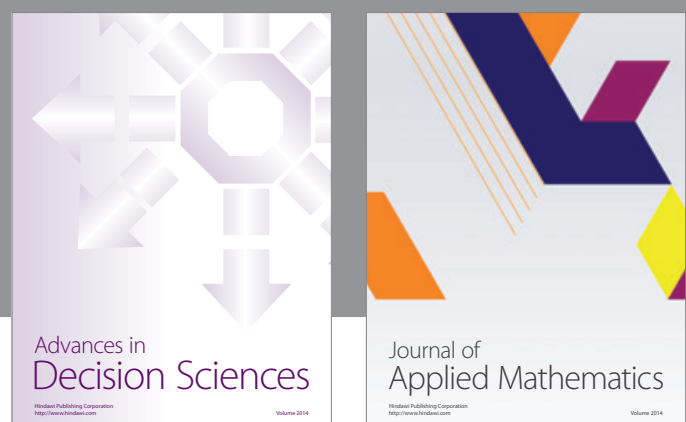

Journal of

Applied Mathematics
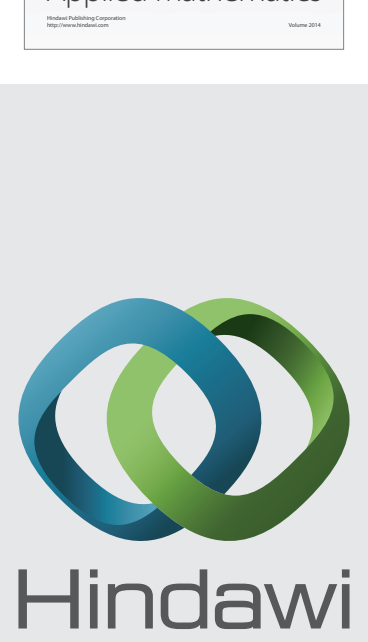

Submit your manuscripts at http://www.hindawi.com
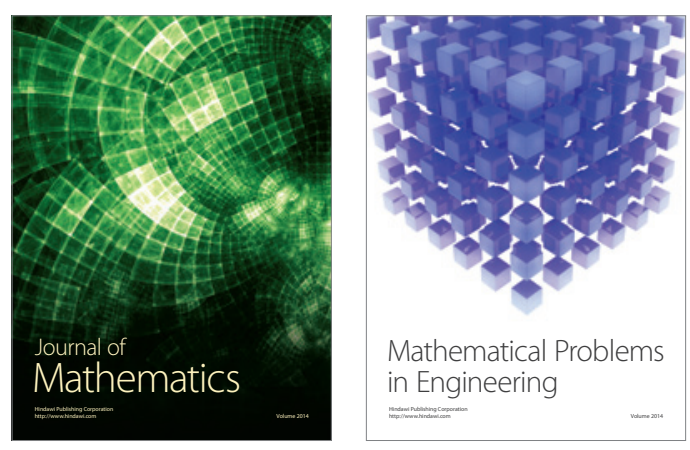

Mathematical Problems in Engineering
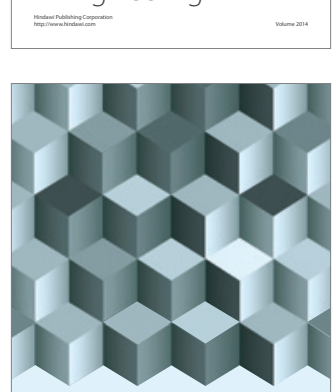

Journal of

Function Spaces
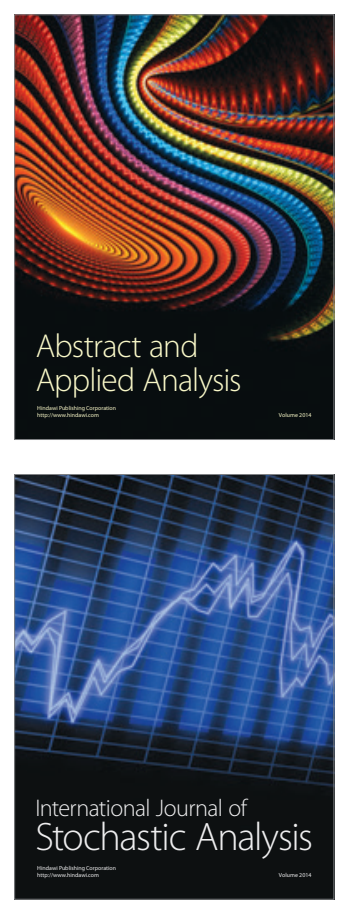

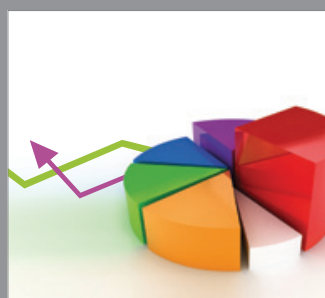

ournal of

Probability and Statistics

Promensencen
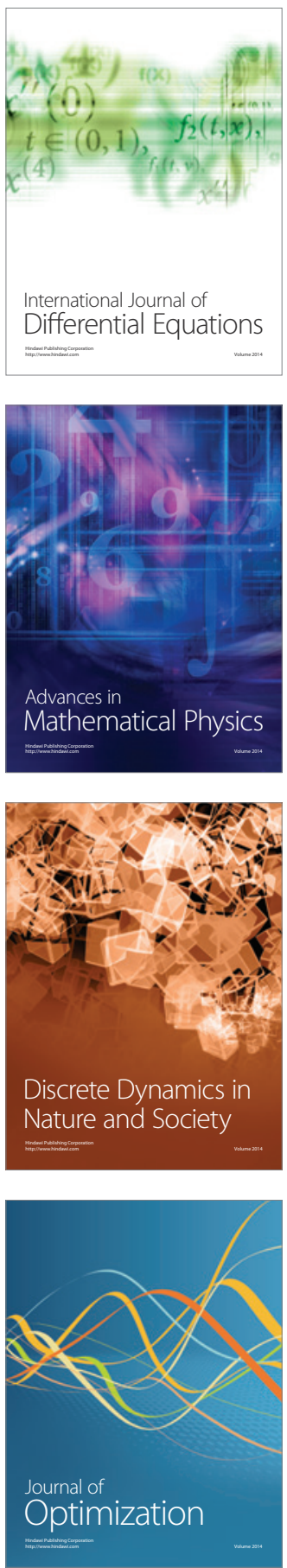